\title{
Brokering Upstream: Providing Information in a Landscape of Health Inequality
}

\author{
Lorayne Robertson \\ University of Ontario Institute of Technology, Canada
}

\begin{abstract}
In Canada, concerns about the level of treatment available for persons with eating disorders have been raised to national attention through a recent parliamentary report which indicates that multiple fronts need attention: including prevention, intervention, and treatment for eating disorders. This predictably places a significant burden of responsibility on health information providers who may be the first point of contact for individuals and families seeking help for an eating disorder, as well as health care practitioners seeking current information. The knowledge broker in this scenario provides current, reliable information about the health issue as well as requisite links to access medical support. This paper examines the theory and practice of health knowledge brokers, focusing in particular on theoretical models which support $e$ health knowledge exchanges and those which involve health equity issues. Findings from this review of the literature indicate that the role of a health knowledge broker in the current era is both enhanced and challenged by e-communication technologies. The collective findings from this ongoing study are expected to shed new light on health mobilization efforts designed to build critical health literacy, advocacy, action, and empowerment in the areas of $e$-health and eating disorders.
\end{abstract}

\section{Introduction}

The review of the literature described in this paper is one segment of a planned investigation of the multiple roles of one health knowledge broker in a not-for-profit Canadian organization which provides a nexus for health information on eating disorders. This organization is also a key point-of-contact for health care professionals seeking information on the prevention, intervention, and treatment of eating disorders. Individuals seeking information from this health knowledge broker might also be in need of contact information for medical treatment for eating disorder-related health concerns. Some of those seeking information may be experiencing a health crisis or they may be seeking information for a family member who appears to be in or approaching a health crisis. Because this health knowledge broker provides a critical service, it is essential that its knowledge brokerage spaces provide timely and accurate information to support the health care of individuals with eating disorders and those close to them. Similarly, if the health knowledge broker disseminates current research to health care providers on the prevention, intervention, and treatment of eating disorders, the health information must meet the same imperatives of currency and accuracy.

While ways of sharing information are not necessarily digital (such as posters, help-phone lines, and conferences) many elements of health knowledge brokering now occur through an online presence such as a website or blog [4]. E-health has emerged in the last 15 years as a major presence - not just as a technological development, but because e-health carries with it an attitude of open communication and the sharing of information [4].

An eating disorders information website also can provide connections to other, similar national and international information centres. It can also provide or host the spaces for sharing information. This has the potential of benefitting not only persons in need of information for urgent care information, but professionals in the fields of health care, social service, and education who work in health care globally. E-health has been defined as the field where medical informatics, health, and business intersect [4].

Because of the serious health implications associated with eating disorders and the reported concerns for rising incidence rates in Canada [6], it makes sense to examine theory and research related to the potential roles which can be undertaken by health information centers, and determine what can be gained through a systematic analysis of present understandings related to knowledge brokering, ehealth, and health equity issues. In particular, the literature here is examined for insights into knowledge brokerage where the terrain of the information is complicated. Research attention on eating disorders is reported within a growing context of rising obesity concerns - combined, these conditions are argued to be the most pressing of 
current health-related issues in the United States [11]. The Canadian eating disorders landscape has some parallels which are outlined in the next section.

\section{Significance of the research}

Eating disturbances are associated with multiple other health problems, and have, unfortunately one of the highest rates of mortality of a mental health disorder [11] in the United States. Despite the fact that disordered eating is a health condition which is under-reported and life-threatening, a recent parliamentary committee [6] heard that there are serious concerns in Canada because of the lack of treatment beds for patients with eating disorders and the wait times for treatment are extensive. The lack of recognition of eating disorder patient needs is considered to be an equity issue because the country is not responding to provide health care for eating disorders with the same urgency as it responds to other potentially fatal illnesses affecting primarily men [6].

Eating disorders affect in the range of $600,000-$ 900,000 persons in Canada, and females are overly at risk; the ratio of females to males is reported to be approximately 4:1 [6]. When considered together, these statistics indicate that there is a need to understand how eating disorder information can be garnered and packaged so that information on current research is shared, and perhaps is delivered with messages which include advocacy to address inequitable health outcomes and health risks for affected Canadians.

This key aspect of the role of the health knowledge broker who reveals inequitable health outcomes and advocates for change has not yet received a significant amount of attention in the literature. Whitehead and Popay [17] utilize the term, "swimming upstream" to describe the nature of work required to reduce health inequalities which are caused by unequal distributions of power and result in unfair and unequal conditions of health care. A knowledge broker for eating disorders in the Canadian context is brokering knowledge upstream to address the needed health care in the face of disparities.

The disproportionate number of girls at risk from eating disorders can be partially explained by examining societal messages which promote the thin ideal body, but explanations as to why there has been an insufficient health care treatment response to eating disorders in Canada are more elusive. The social pressure to be a certain size in Canada puts girls and women at risk of eating disorders, especially if they identify their acceptance in society with their appearance [13].
Canada has a strong consumer culture which advocates purchasing products to improve one's appearance. The unrealistic and often unattainable mediated thin ideal makes the work of an eating disorders information center more difficult. Messages about body acceptance (designed to discourage people from undertaking body-altering diets) are required, but their promotion is almost counter-cultural (like swimming upstream) next to ubiquitous media messages focused on body improvement. The health field itself is concerned with rising rates of obesity, and this, as well, complicates the health landscape with weight and size bias.

Within this culture, messages of accepting body size rather than changing body size can be challenging to broker. The health knowledge broker in this scenario is working in a national context of a (real or mediated) obesity crisis, while concurrently brokering messages of health at every size to both the medical community and the average Canadian. Again, little has been written about health knowledge brokers who undertake their work in this type of contested terrain. Regardless of where one might stand philosophically on these issues, when the incidence of disordered eating is reported to be almost matched by the obesity incidence [11], it is evident that knowledge translation of current research on these issues is urgently needed. In summary, then, within this complicated social scenario of responding to issues of health and size, a health information provider needs to consider carefully how it will represent this divide in health knowledge positions to their information users. In this scenario the knowledge broker must decide which health knowledge it will advance and support.

A second issue is health equity. In the case of eating disorders, there is a reported disparity of impact for women in Canada. In the face of unequal, unjust or unfair health outcomes, a health equity stance can push for more accountability in both programs and policy to redistribute healthy outcomes [1]. As Braveman and Duskin explain, groups of people defined by their differences are more advantaged or less advantaged in a social hierarchy. The markers of inequality such as income, race, religion, gender, geography, age, disability, sexual orientation (and others) are reflected in health inequalities around the world [1]. A health disparity occurs when a group which is already marginalized in society is further marginalized in the health forum. Health equity is reflected in the re-allocation of resources and processes to correct these inequities [1]. In this scenario, the role of the knowledge broker needs to be theorized to include awareness and advocacy to address identified health inequities. 
The specific case of eating disorder prevention, intervention, and treatment in the Canadian context resembles other, similar cases which are complex, multi-layered, and complicated. A health information provider needs to provide the means to inform knowledge users but also cannot ignore health inequities. In the face of these challenges, the health knowledge provider may choose to build the capacity of the knowledge users. These capacity-building efforts would go beyond providing information to encouraging them to be discerning consumers of research information, and empower them to make informed health decisions. In this scenario, the health knowledge worker builds capacity for critical understandings, and provides not only information, but builds empowerment.

Once again, this type of health knowledge brokering is an area of research that is underinvestigated and needs to be explored more fully.

\section{Theoretical Perspectives}

The understanding of the role of the knowledge broker is enhanced through an analysis of relevant theories which help to explicate the roles of knowledge brokers, the boundaries of the arenas from which they operate, and some of the imperatives which underlie their commitment to brokering the spaces between research and practice, or research and the public. Theoretical perspectives on knowledge brokering have evolved from earlier (less effective) models where research was passively disseminated from the academy to more active dissemination efforts; it was at this point that role of the knowledge broker emerged [15]. First, theoretical perspectives relative to knowledge brokering are discussed, followed by a theoretical discussion about the importance of health equity in post-modernity.

\subsection{Knowledge brokering}

The role of the health knowledge broker has recently begun to receive more attention through research and scholarship, and new models are emerging. Early studies of knowledge brokering identified four models for the dissemination of science research knowledge: knowledge that was pushed out from the researchers to the consumers, knowledge which was pulled or requested by the users, knowledge which was disseminated by the researchers through journals for example, and knowledge which was brokered through interactions between researchers and users [6]. Of these four models, the brokered, interactive model of sharing knowledge between researchers and their audience has shown the most potential for knowledge sharing [6]. Knowledge brokers are seen as go-betweens or intermediaries who bridge the worlds of research and practice and make each side of the divide more accessible to the other [15]. Research which is disseminated passively has less chance of uptake than research which has been commissioned, or research which has been actively disseminated and translated using summaries, slides and handouts, or also facilitating events where knowledge is shared etc. [15]. For this, knowledge brokers need excellent communication skills and understandings of both policy issues and research findings. What is significant about the research on knowledge brokers is that there is little evidence demonstrated to date that the knowledge broker can promote more equitable relationships between the researchers and those who make policy decisions. In addition, evaluations of knowledge brokerages which are focused on capacity building and information literacy are to date mostly anecdotal rather than scientific [15].

Graham et al. [5] argue that health knowledge transfer can be a slow process, which has implications if there is a time lag between the publication of the research findings (knowledge creation) and when the research is put into place to benefit the patient (knowledge application). In their exploration of this phenomenon, one of the issues cited is the confusion of terminology. They located 29 terms used to describe the knowledge to action concept [5]. They propose a theoretical model of moving knowledge into action, beginning with problem identification, the selection of knowledge relevant to the problem, adaptation of the knowledge for the user, assessment of barriers, the actual knowledge intervention, and then the monitoring of the knowledge use and outcomes. Their model places a focus on identification of the needs of the users and the effective dissemination of knowledge monitored through continuous feedback loops [5].

There are, however, several emergent and promising frameworks which theorize the role of the knowledge broker with a post-modern outlook of shared knowledge creation. Meyer, for example, looks at the spaces where knowledge is exchanged, and acknowledges that while some spaces (e.g., a university technology transfer department) are more privileged in the transfer of knowledge, other exchanges are happening behind the scenes which could be characterized as more collective, and with more interactions. She theorizes that knowledge is often exchanged at the peripheries or boundaries between the world of the researchers and the intended audiences who utilize the research. [8]. Knowledge brokers who are marginally connected to both the 
world of research and that of the knowledge user can operate between them and in both worlds without being tied to the philosophy of one domain over the other. This is seen as an advantage despite the fact that academic knowledge continues to attract more attention. Knowledge brokers who work in this way at the peripheries are also seen to function more or less behind the scenes. Meyer sees that this invisibility can have its advantages, allowing the knowledge broker to move back and forth between different worlds while functioning in each [8].

Some of the tasks of the knowledge broker include the identification of the significant knowledge to disseminate, then the re-scaling of this knowledge in order to redistribute it to the knowledge users [8], a process sometimes called knowledge translation [5]. The knowledge broker has to know how to re-package the information based on the identified needs of the research audience [5], [8]. According to Meyer, in this process, the knowledge broker does not simply transmit but transforms the knowledge so that it can be taken up most effectively. The knowledge which is the end product of these partnerships is more robust because it has been deconstructed and then reconstituted for knowledge transfer [8].

According to Ward et al., [15], knowledge brokering can fall into one of three types - managing information, building linkages, and/or capacity development. Key roles of the knowledge broker include the ability to gather information, critically assess it, synthesize it, and tailor it to the audience. To that end, communication skills, mediation skills, networking skills and the ability to gain credibility are very important. The knowledge broker needs to focus on relationships and partnership development to keep both knowledge creators and knowledge users engaged in productive partnerships. For all of these reasons, knowledge brokering needs to be conceptualized as a complex social endeavour [15].

\subsection{E-Health}

Health services and health information can now be accessed and delivered via the internet, leading to a new field of study and new possibilities for persons seeking medical advice, health care providers, and health care information providers. Eysenbach has defined the concept of e-health as "an emerging field in the intersection of medical informatics, public health and business, referring to health services and information delivered or enhanced through the Internet and related technologies" [4]. He makes the distinction that e-health is not just about the technology or the enhanced provision of information, because e-health is also an attitude or a state of mind which focuses on improving health care through networked thinking. While he acknowledges that ehealth has aspects of efficiency, he also sees that ehealth has the potential also for empowerment because the knowledge bases of medicine are now open to the public, and users of e-health information can become more critical consumers. He cautions, however, that the digital divide might further exacerbate equitable access to health unless policies allow for e-health access for all [4].

Skinner and colleagues [13] examined how adolescents in Ontario, Canada use technology to access health information, finding that adolescents do need health information and they tend to use both technological and traditional sources to access that information. A significant finding of their study was that the adolescents most commonly sought information about specific medical conditions and diseases (67\%), followed closely by the search for information about body image and nutrition (63\%). Based on this study, health care providers can create a "major interface" with adolescents seeking health information through technology. Technology could accomplish this through extending the times and means by which online health support is available, and finding ways to make appear to be more approachable to adolescents [13].

The Skinner et al., study [13] also found that adolescents employ different technologies based on the situation and their needs. For example, cell phones were most often used for personal safety, including the use of toll-free numbers to access specific help. The adolescents considered email to be the most accessible technology, particularly if their health condition was one that they found difficult to discuss in person. Adolescents saw bulletin boards and chat rooms as helpful for obtaining more specific information in these instances. A significant finding in this study was the adolescents indicated that they were overwhelmed with information on the internet, especially when they wanted an answer for a specific question, and they needed support with finding reliable sources. Adolescents also indicated that they preferred online sources rather than traditional sources of health information for situations where the health concern might cause embarrassment [13].

It is not only adolescents who eschew traditional sources of medical information for the internet, however. Another key user group for internet health information is persons who have stigmatized illnesses. This stigma can lead them to feel embarrassed and want to conceal their health condition - they might delay seeking health care and instead, turn to the internet for information. Persons who perceive a stigma surrounding eating disorders can be more reluctant to talk about their problem with 
medical practitioners and this can delay seeking treatment.

Roehrig and McLean [12] find that eating disorders have been shown to be "highly stigmatized" because there are perceptions that persons with eating disorders are to blame for their illness, or may be acting to get attention, or that they are somehow choosing to be ill. The issue becomes complicated by public messages of normalcy surrounding weight and shape pre-occupation, while holding a stigmatized view surrounding those to have related illnesses. In addition, adolescent envy of persons with eating disorders further complicates the stigmatization of eating disorders [12].

What can be learned from these specific studies and other related studies on internet use and stigmatized illnesses is that the provision of e-health options for persons with eating disorders is more than a communication option for health knowledge brokers - it is an imperative. Much more study is needed to understand how persons with stigmatized illnesses use technology for personal safety reasons and to seek medical information. The provision of accurate, timely and accessible health information has the potential to build skills of critical health literacy. The connections between health equity and health literacies are explored next.

\subsection{Health equity and critical health literacy}

A theoretical framework of health equity is helpful in exploring the work of knowledge brokers who support a group of people who have been historically-disadvantaged based on evidence of systemic disparities in in health treatment availability and outcomes. Braveman and Gruskin [1] define equity in health as "the absence of systematic disparities in health...between social groups who have different levels of underlying social advantage/disadvantage" (p. 254). Similarly, in a World Health Organization report, Whitehead and Dahlgren [16] describe health inequities as "unfair and unjust" (p. 5). They see that health equity means that no person should be disadvantaged from achieving their full health potential because of their position in society or any other "socially determined circumstance" (p. 5).

In the specific circumstance of persons who are afflicted with eating disorders, there are multiple areas of inequity. Research indicates that persons with eating disorders face discrimination in society. The social stigma attached to disordered eating increases isolation rather than encouraging a person to disclose what they suspect about their condition and seek medical attention. In addition, significant media attention is placed on persons who have thin, sculpted bodies with little attention directed at the ways that they have altered their eating and exercise regimens to achieve an appearance that is essentially not their natural shape. The issue is further confused by mediated circumstances where extreme weight loss is connected to the pursuit of health. Mediasupported celebrations of extreme weight loss collide with the real needs of persons for whom extreme weight loss is an indication of illness.

Discrimination is another form of inequity which is realized through barriers to effective health treatment. For example, persons with eating disorders are discriminated against in society because there is a perception that their behaviours and choices cause their medical problem (self-attribution). This social stigma is not only harmful for the self-esteem of persons seeking help, it also inhibits people with eating disorders from seeking the attention of medical professionals [12]. In order to address discrimination and stigma, it is important to educate many groups: persons with eating disorders, health care practitioners, and the general public.

One key strategy which shows promise is to empower and enable persons seeking medical attention to discuss and disclose their symptoms and seek help is the development of critical health literacy skills. Nutbeam, for example, sees critical health literacy as a key means of promoting the health of marginalized groups through empowerment and education. He argues that earlier forms of health education focused on telling people what to do or not do while using the transmission of knowledge to do this. He finds these methods to be less effective than building capacity or building health literacies. $\mathrm{He}$ defines critical health literacy as a level of advanced skills which can critically examine information, then encourage a person to take a more active role in her/his health [10].

Implicit in a commitment to critical health skill development is an attitude of providing the requisite information as well as the skills to help people understand research knowledge in ways that the knowledge becomes accessible to them. It also means that the health information provider does not hide the issues of unequal treatment and societal discrimination but includes them in building critical health literacy skills. This requires encouraging a questioning stance toward the research and applying levels of analysis to help the consumers of health information understand that there are issues of power associated with whose knowledge is promoted, who gets more help when they are ill, and where the focus is placed nationally in prevention, intervention and support for certain medical conditions.

The view of the health information consumer as a passive recipient of health information is gradually 
being replaced by considerations of how literate persons interact with health information. When critical health literacies are advanced, individuals are less likely to assign self-blame and shame and understand conditions of illnesses which are outside of their individual control. In addition, a critical health literacy stance is one which has openness to accessing information and encourages knowledge users to add levels of analysis to the information and use it to empower them to take action. As a result of these findings in the literature, Nutbeam's theory of critical health literacy [10] influences the design of this study and the data analysis.

\section{Methodology}

The research which is proposed will fully explore the experiences and voices of persons who work in a health knowledge broker environment, including those who partner with the health knowledge broker in multiple endeavours. The planned research is phenomenological in its perspective according to parameters outlined by Cresswell [3]. As such, the research will seek to understand the everyday lives of persons who work for one national health information centre which provides information on eating disorders. Also included in the investigation will be their web spaces and online materials.

This review of the literature indicates that adolescents employ different technologies to seek information and support for their health needs [13]. Murphy, Frost, Webster, and Schmidt [9] examined international websites intended to provide information on eating disorders. Their findings suggest that there is significant room for improvement and request more connections for knowledge exchanges between health professions and authors of materials on the web. Murphy et al., also find that financial considerations impact the development of web spaces [9]. For these reasons, the web space will be included in the planned data collection as well as all other aspects of knowledge brokering which employ technology such as call centers and other phone-in services.

Specifically, this research is intended to uncover the ways that one particular knowledge broker meets the needs of multiple participants in the knowledge brokering activities, including knowledge users, knowledge broker partners and relationships, and knowledge producers from different area such as research and health care policy. The study will attempt to identify the categories of constituents and seek specifically to examine the knowledge interchanges and brokering spaces where health research and information is shared through this investigation.

\section{Findings}

The review of literature for this planned study revealed that there are several key theoretical frameworks which will help to design the study and analyse the findings. The first is current research on health knowledge brokers. The second will be theoretical considerations surrounding the provision and brokering of e-health information. The third theoretical consideration is that of critical health literacy to address an area of health information that has been shown to receive unequal treatment and health outcomes.

With respect to the first area, knowledge brokering, this literature review indicates that there are multiple terms in use with respect to knowledge brokerage, such as knowledge exchange, knowledge transfer, knowledge translation, and knowledge mobilization (an others). There are also many activities and initiatives which take place under the umbrella of health knowledge brokerage. In addition to examining knowledge brokering processes, however, it is important to examine the spaces where health knowledge brokering occurs and how health knowledge brokers set about to create those spaces.

The review of the literature has added the consideration for the planned study on the topic of relationships in health knowledge brokering. According to the Canadian Health Services Research Foundation (CHSRF) [2], "Knowledge brokering is about bringing people together to help them build relationships, uncover needs, and share ideas and evidence that will let them do their jobs better" (p. 1). This foundation also maintains that much of the present knowledge brokering is unrecognized activity which needs to be documented carefully through research [2].

The second theoretical consideration raised in the literature is that of e-health information systems. Again, while the research in this area is at a nascent stage, there are some indications that access to health information using technologies is particularly suited to providing support where the illness has a perceived stigma which presents a barrier to seeking support and early diagnosis through traditional means.

The third theoretical construct to support the research is the study of the development of critical health literacy through the efforts of a health knowledge broker. In the post-modern era of open access to information and challenges to taken-forgranted power structures (such as gender-based disparities in health outcomes) critical health literacy has emerged as a key concept for empowerment and action.

In summary then, this review of the literature indicates that more research is needed on eating 
disorders and understanding the role of knowledge brokers relative to this illness. In addition, gaps were identified with respect to the roles of health knowledge brokers in situations where there are health disparities of outcomes. Third, there is a gap in the research in understanding processes, actors and spaces for the development of critical health literacy. A case study of one health knowledge broker who focuses in the area of eating disorders is designed to build understanding in these areas and encourage other, similar research.

\section{Recommendations}

While the research reported here is at a beginning stage, the review of the literature indicates that the planned research to investigate the roles, spaces and relationships provided by one health care knowledge broker will fill a gap in present understandings of the roles of health knowledge brokers. As this research progresses, it will not only provide information on health knowledge brokering in general, but health knowledge brokering in particular in the Canadian health sciences context. It will, in addition, provide more information on health knowledge brokering that takes action to address health inequities. The phrase knowledge brokering upstream captures some of the essence of the struggle to provide health information in an area which is experiencing unequal health outcomes in the present Canada context.

\section{References}

[1] Braveman, P., \& Gruskin, S., (2003). Defining equity in health. Journal of epidemiology and community health, 57(4), 254-258.

[2] CHSRF, (Canadian Health Services Research Foundation). (2003). The theory and practice of knowledge brokering in Canada's health system. Ottawa, ON,Canada: Author.

[3] Creswell, J. W., (2012). Qualitative inquiry and research design: Choosing among five approaches. Sage.

[4] Eysenbach, G., (2001). What is eHealth. Journal of Medical Internet Research, 3(2), e20.doi:10.2196/jmir.3.2. e20.

[5] Graham, I. D., Logan, J., Harrison, M. B., Straus, S. E., Tetroe, J., Caswell, W., \& Robinson, N., (2006). Lost in knowledge translation: time for a map? Journal of continuing education in the health professions, 26(1), 1324.

[6] House of Commons, (2014). Eating disorders among girls and women in Canada: Report of the standing committee on the status of women. Canada: House of Commons. Retrieved @ http://www.parl.gc.ca/HousePubli cations/Publication $\cdot$ aspx $?$ DocId $=6772133 \&$ Mode $=1 \&$ Parl $=$ $41 \&$ Ses $=2 \&$ Language $=\mathrm{E}$.

[7] Landry, R., Amara, N., \& Lamari, M., (2001). Utilization of social science research knowledge in Canada. Research policy, 30(2), 333-349.

[8] Meyer, M., (2010). The rise of the knowledge broker. Science Communication, 32(1), 118-127.

[9] Murphy, R., Frost, S., Webster, P., \& Schmidt, U., (2004). An evaluation of web-based information. International Journal of Eating Disorders, 35(2), 145-154.

[10] Nutbeam, D. (2000). Health literacy as a public health goal: a challenge for contemporary health education and communication strategies into the 21 st century. Health.

[11] Smolak, L. \& Thompson, K., (2009). Body image, eating disorders, and obesity in children and adolescents. Introduction to the second edition. In L. Smolak \& K. Thompson (Eds.), Body image, eating disorders and obesity in youth: Assessment, prevention and treatment (pp. 3-14). Washington, DC: American Psychological Association.

[12] Roehrig, J. P., \& McLean, C. P., (2010). A comparison of stigma toward eating disorders versus depression. International Journal of Eating Disorders, 43(7), 671-674.

[13] Robertson, L. \& Thomson, D., (2012). "Being a certain way: Seeking Body Image in Canadian health and physical education curriculum policies. Canadian Journal of Education, 35 (2), 334-354. Canadian Society for the Study of Education.

[14] Skinner, H., Biscope, S., Poland, B., \& Goldberg, E., (2003). How adolescents use technology for health information: implications for health professionals from focus group studies. Journal of medical internet research, $5(4)$.

[15] Ward, V., House, A., \& Hamer, S., (2009). Knowledge brokering: the missing link in the evidence to action chain? Evidence \& policy: a journal of research, debate and practice, 5(3), 267.

[16] Whitehead, M., \& Dahlgren, G., (2006). Concepts and principles for tackling social inequities in health: Levelling up Part 1. World Health Organization: Studies on social and economic determinants of population health, (2). http://www.pacifichealthsummit.org/downloads/Obesity\%2 OPrevention \%20and $\% 20$ Control\%20Efforts\%20in\%20Sing apore $\% 20-\% 202008 \% 20$ Case $\% 20$ Study.pdf.

[17] Whitehead, M., \& Popay, J., (2010). Swimming upstream? Taking action on the social determinants of health inequalities. Social science \& medicine, 71(7), 12341236. 